\title{
Rare occurrence of cavitation of lung metastases following effective targeted therapy: A case report
}

\author{
JIACHENG SONG, JING YU, ZHANLONG MA and SHANSHAN LU
}

Department of Radiology, The First Affiliated Hospital of Nanjing Medical University, Nanjing, Jiangsu 210029, P.R. China

Received September 22, 2014; Accepted November 24, 2015

DOI: $10.3892 / \mathrm{ol} .2016 .4093$

\begin{abstract}
The metastasis of malignant tumors to the lung is relatively common; however, cavitation of metastases is extremely rare in clinical cases, and the various mechanisms are controversial. The majority of irregular metastatic cavities form naturally and are detected incidentally when patients are examined prior to any therapeutic measures. The current study presents the case of a lung adenocarcinoma patient with total cavitation of intrapulmonary metastases following effective targeted therapy at the First Affiliated Hospital of Nanjing Medical University. The morphological changes were followed up for three years. This patient presented thin-walled and smooth clean cavities, which provides an indication of the effectiveness of treatment.
\end{abstract}

\section{Introduction}

Approximately $4 \%$ of metastases in the lung evolve into cavity lesions, two-thirds of which are from squamous cell primary tumors. Spontaneous cavitation of metastases from the bladder (1), kidney (2) and synovial sarcoma (3) have been reported, however, few studies have reported the cavitation of metastases following systematic treatment (4). Post-therapy cavitation from gland cell primary tumors is particularly rare, and we hypothesize that the cavitation process following therapy involves a completely different mechanism and morphology compared with the necrotic tumor process.

Currently, the incidence of non-squamous non-small-cell lung cancer (NSCLC) is increasing and the disease is frequently associated with the activation of genetic alterations that become innovative therapeutic targets (5). This case report describes the cavitation of multiple solid lung metastases in targeted monotherapy in a 39-year-old male, and follows the changes for three years. The patient provided informed consent for the publication of this study.

Correspondence to: Professor Zhanlong Ma, Department of Radiology, The First Affiliated Hospital of Nanjing Medical University, 300 Guangzhou Road, Nanjing, Jiangsu 210029, P.R. China

E-mail: mazhanlong@126.com

Key words: adenocarcinoma, mutation, cavitating metastases

\section{Case report}

Case presentation and treatment. A 39-year-old male presented at The People's Hospital of Jingjiang (Jingjiang, China) in December 2007 with the complaint of a cough with left-side chest pain for four years and sputum with blood for a week. He was a non-smoker, but had smoked passively for five years. Chest computed tomography (CT) was normal. In 2011, the constant stuffy pain had gradually increased without cause and the patient spitted sputum with blood streaks after playing badminton. Local hospital chest radiography revealed a lesion occupying the left lung, and the patient was admitted to the Respiratory Department of the First Affiliated Hospital of Nanjing Medical University (Nanjing, China), for further investigation. Abnormal tumor markers revealed a carcinoembryonic antigen level of $13.69 \mathrm{ng} / \mathrm{l}$ and a neuron-specific enolase level of $18.29 \mathrm{ng} / \mathrm{l}$. In October 2011, chest CT revealed a left upper lobe stiff spiculated mass with multiple intrapulmonary nodules (Fig. 1). A left lower pulmonary wedge resection was conducted following consultation with the Cardial Surgery Department, and the pathological result was adenocarcinoma (T2aN3M1a-IV). Further gene mutation detection presented epidermal growth factor receptor (EGFR) gene mutation with exon $21 \mathrm{~T} / \mathrm{G}$ hybrid positivity, which prompted the clinical use of EGFR gene-targeting drugs. The patient's Karnofsky score was 90, and Tarceva was administered orally on a daily basis. During the process, regular chest CT and tumor markers were monitored.

3-year follow-up. In December 2011, a chest CT scan revealed the primary tumor together with multiple metastases appearing to be decreased in size and of a relatively lower density. Certain metastatic nodules were round with a small hole in the center.

In June 2012, chest CT revealed further improvement of the primary tumor and metastases. Multiple irregular cavities with thickened septum appeared to have replaced the original tumor node metastases. The patient's carcinoembryonic antigen level was $7.04 \mathrm{ng} / \mathrm{l}$.

In August 2013, chest CT demonstrated larger and clearer cavities resembling pulmonary bullous, while the primary cancer increased in size. In the period that followed, the patient's tumor markers and chest CTs were monitored every two months until April 2014. The patient's carcinoembryonic antigen level increased to $9.9 \mathrm{ng} / \mathrm{l}$ and he experienced waist pain. Lumbar magnetic resonance imaging revealed 


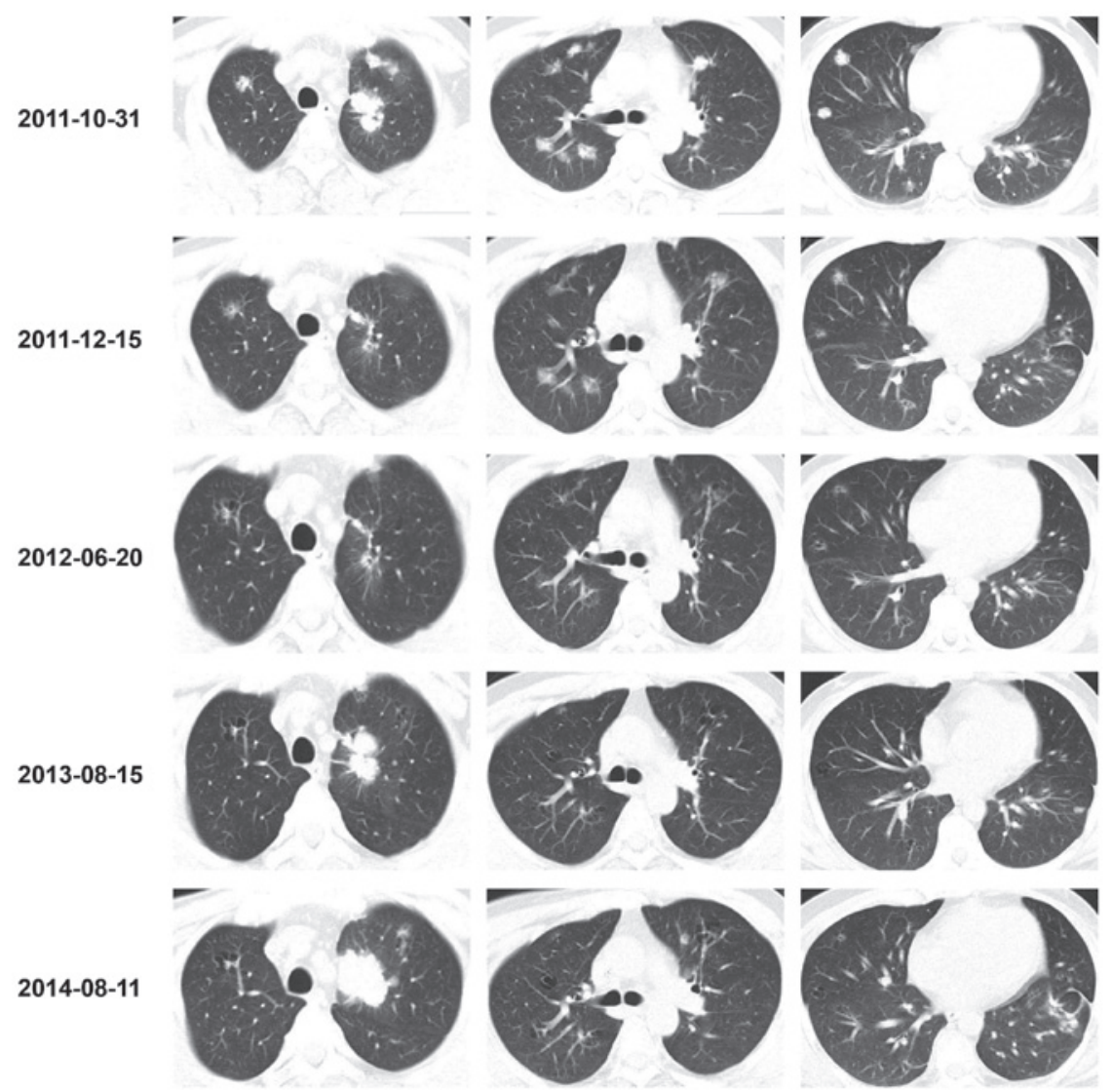

Figure 1. Presentation and evolution process of niduses on chest computed tomography at various points of the follow-up period.

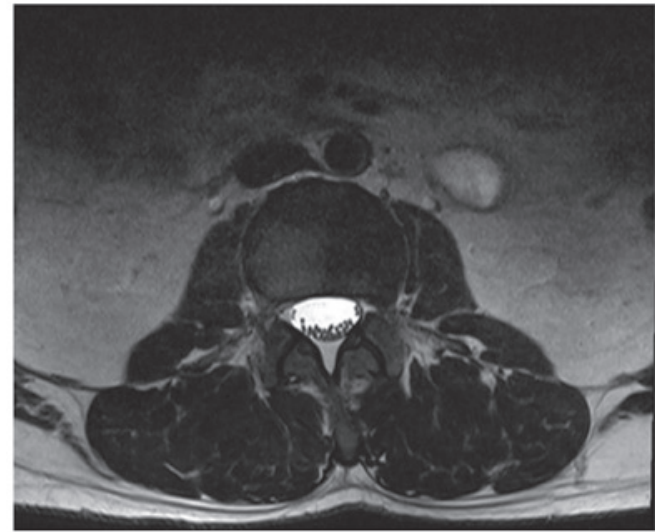

Figure 2. Lumbar magnetic resonance imaging reveals bone metastasis with a hyperintense nodule in the third lumbar vertebrae on T2-weighted image.

a hyperintense nodule in the third lumbar vertebrae on the T2-weighted image (Fig. 2). The pathological result following surgery confirmed the suspicion of bone metastasis. The patient started a chemotherapy regimen of pemetrexed disodium $(0.9 \mathrm{~g})$ plus carboplatin $(600 \mathrm{mg})$ once a month.

In August 2014, during the fourth month of chemotherapy, the patient had a carcinoembryonic antigen level of $9.7 \mathrm{ng} / \mathrm{l}$, a cytokeratin 19 fragment level of $0.7 \mathrm{ng} / \mathrm{l}$ and a neuron-specific enolase level of $14.4 \mathrm{ng} / \mathrm{ml}$. Chest CT revealed the growth of the original tumor with several new metastases and slight pleural effusion on the left side, without any notable changes to the existing cavities.
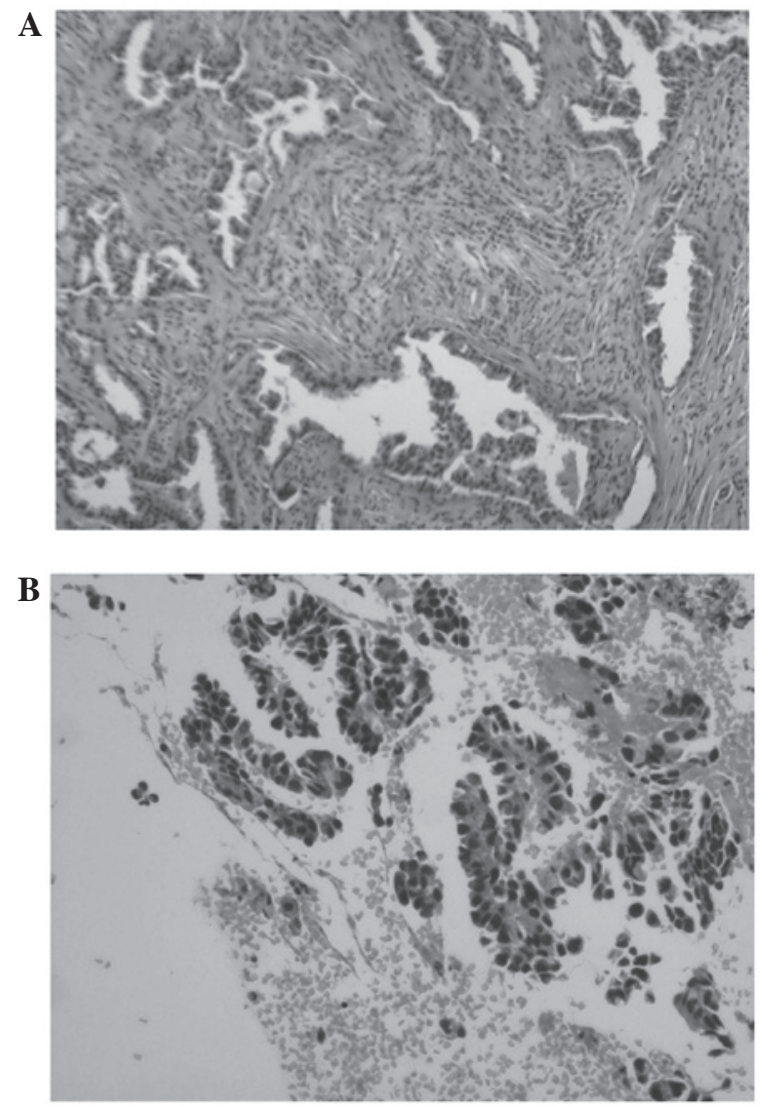

Figure 3. Microscopic images of lung and vertebral metastases reveals glands arranged dysplastic cells. Hematoxylin and eosin staining; magnification, x100 (A) and x200 (B). 
Pathological analysis. The pathological results of the surgical specimen revealed dysplastic cells arranged in a gland-like formation among the intercellular matrix, which presented a microscopic manifestation typical of adenocarcinoma (Fig. 3). Papillary arrangements of broken atypical epithelial cells were noted among the bone tissue and blood cells on the vertebral pathological analysis (Fig. 4).

\section{Discussion}

Outcomes for locally advanced NSCLC remain suboptimal, with a median overall survival duration of 20 months (6). The identification of somatic mutations of EGFR brought about a paradigm shift in therapeutic strategy (7). Extensive research focused on the survival outcome of patients with EGFR-mutant advanced NSCLC depending on the different combined modality therapy employed or whole-body extent of metastasis $(8,9)$. In the present study, we presented a rare case of cavitation transition of lung metastasis owing to effective targeted therapy. Although in the later stage of the disease process, the primary tumor turned to a growth momentum and new metastases appeared, the already formed smooth cavities did not demonstrate notable changes and never filled with fluid or necrotic debris.

Cavitation usually forms as a result of necrosis from insufficient blood supply to the tumor or invasion of vessels by tumor cells, which always raises the likelihood of disease progress. Kurian et al (10) reported a case of urothelial bladder cancer with cavitary lung metastases, which exhibited a large irregular cavity surrounded by a wall of uneven thickness. This type of cavity is formed by central ischemic necrosis with the progression of the tumor. Unlike common cavities with irregular lumen and wall nodules, this type of thin-walled and smooth morphology may reflect a completely different mechanism and to a certain extent supports the improved treatment effect. Mermershtain et al (4) reported the only case of massive cavitation of solid pulmonary metastatic lesions in a breast cancer patient following adjuvant chemotherapy and radiation therapy. The regular and sharply defined cavities resemble those of our case and may specifically appear following effective therapy.

For the present case, this type of regular cavitation resulted from inhibited tumor angiogenesis and provided clinical reference value for treatment effectiveness.

\section{References}

1. Koh KB, Rogawski K and Smith PH: Cavitating pulmonary metastases from superficial transitional cell carcinoma of urinary bladder. Case report. Scand J Urol Nephrol 28: 201-202, 1994.

2. Essadki O, Chartrand-Lefèbvre C, Finet JF and Grenier P: Cystic pulmonary metastasis simulating a diagnosis of histiocytosis $\mathrm{X}$. J Radiol 79: 886-888, 1998 (In French).

3. Cohen MC and Drut R: Persistent interstitial pulmonary emphysema-like cyst associated with metastatic synovial sarcoma. Pediatr Dev Pathol 3: 391-393, 2000.

4. Mermershtain W, Schulman H, Hertzanu Y and Cohen Y: Massive cavitation of solid pulmonary metastatic lesions in a breast cancer patient: A case report. Ann Oncol 13: 173-174, 2002.

5. Wu M, Zhao J, Song SW, Zhuo M, Wang X, Bai H, Wang S, Yang L, An T, Zhang Y, et al: EGFR mutations are associated with prognosis but not with the response to front-line chemotherapy in the Chinese patients with advanced non-small cell lung cancer. Lung Cancer 67: 343-347, 2010.

6. Hanna N, Neubauer M, Yiannoutsos C, McGarry R, Arseneau J, Ansari R, Reynolds C, Govindan R, Melnyk A, Fisher W, et al; Hoosier Oncology Group; US Oncology: Phase III study of cisplatin, etoposide, and concurrent chest radiation with or without consolidation docetaxel in patients with inoperable stage III non-small-cell lung cancer: the Hoosier Oncology Group and U.S. Oncology. J Clin Oncol 26: 5755-5760, 2008.

7. Azzoli CG, Baker S Jr, Temin S, Pao W, Aliff T, Brahmer J, Johnson DH, Laskin JL, Masters G, Milton D, et al; American Society of Clinical Oncology: American Society of Clinical Oncology Clinical Practice Guideline update on chemotherapy for stage IV non-small-cell lung cancer. J Clin Oncol 27: 6251-6266, 2009.

8. Lee DS, Kang JH, Lee CG, Kim SJ, Choi YJ, Lee KY and Kim YS: Predicting Survival in Patients with Advanced Non-squamous Non-small Cell Lung Cancer: Validating the Extent of Metastasis. Cancer Res Treat 45: 95-102, 2013.

9. Mak RH, Doran E, Muzikansky A, Kang J, Neal JW, Baldini EH, Choi NC, Willers H, Jackman DM and Sequist LV: Outcomes after combined modality therapy for EGFR-mutant and wild-type locally advanced NSCLC. Oncologist 16: 886-895, 2011.

10. Kurian A, Lee $\mathrm{J}$ and Born A: Urothelial bladder cancer with cavitary lung metastases. Can Respir J 18: e46-e47, 2011. 